\title{
36 años de la revista Ería
}

$E^{\prime}$ n el año 1980 veía la luz el primer número de la revista Ería; algo más de tres décadas y media después alcanzamos el centenar de publicaciones, con lo que se cierra una primera etapa de la revista, al tiempo que se abre una nueva, que sin perder el espíritu inicial pretende incorporar Ería a los nuevos formatos de difusión de las revistas científicas.

Parece un buen momento, pues, para hacer un balance, mirar el camino recorrido y marcar unos objetivos para la nueva etapa. Los datos globales no dejan de ser significativos: 525 artículos publicados, con un total de 644 autorías, 80 notas, además de 128 reseñas y 219 recensiones ${ }^{1}$.

Pero detrás de estas cifras frías está una trayectoria que conviene repasar. En la presentación del primer número, el profesor don Francisco Quirós, fundador y director de la revista a lo largo de los 36 años de vida de la misma (honorífico desde el número 84), exponía de una forma clara el sentido de la aparición de Ería y el fundamento de su nombre:

[...] aunque el número de profesores de nuestra Sección no es, ni de lejos, el necesario para hacer frente a las necesidades, sí es ya suficiente como para que el resultado de sus trabajos de investigación, y el de los alumnos, requiera un cauce periódico de comunicación. Con esa finalidad, y también con la de ser útil, como medio de expresión, a compañeros de otros centros, comenzamos a publicar esta revista. En cierto modo, a esta intención de partida de servir de instrumento colectivo responde el nombre que para ella hemos elegido, pues ERÍA es término con el que se designaba en algunos lugares de la España cantábrica a un conjunto de tierras de labor, de propiedad individual, agrupadas bajo una cerca colectiva.

\footnotetext{
${ }^{1}$ La elaboración de los datos de la revista para esta nota ha sido realizada por Salvador Beato Bergua, Ícaro Obeso Muñiz y Héctor Rato Martín, becarios de investigación del Departamento de Geografía.
}

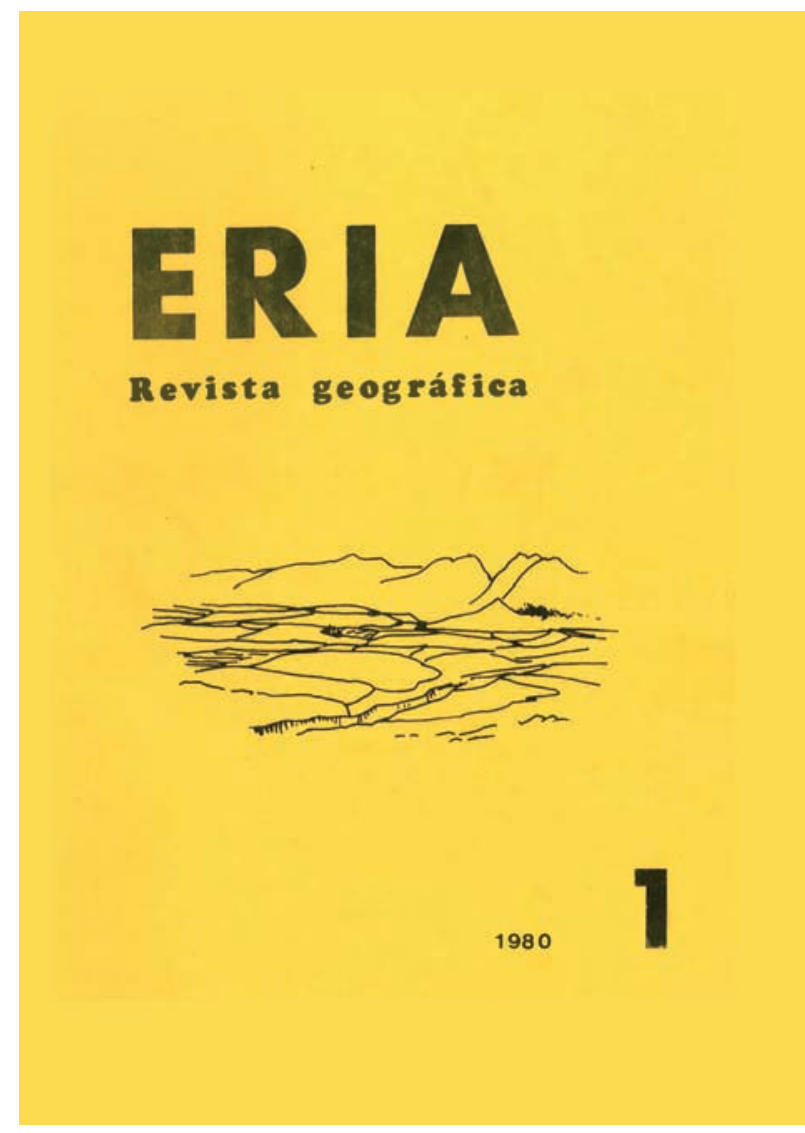

Portada del primer número de la revista.

El nombre no es, pues, un asunto baladí; encerraba y encierra un significado, al referirse a la inserción de la labor individual de los investigadores en un marco de referencia de carácter colectivo. Pero Ería tuvo desde el 


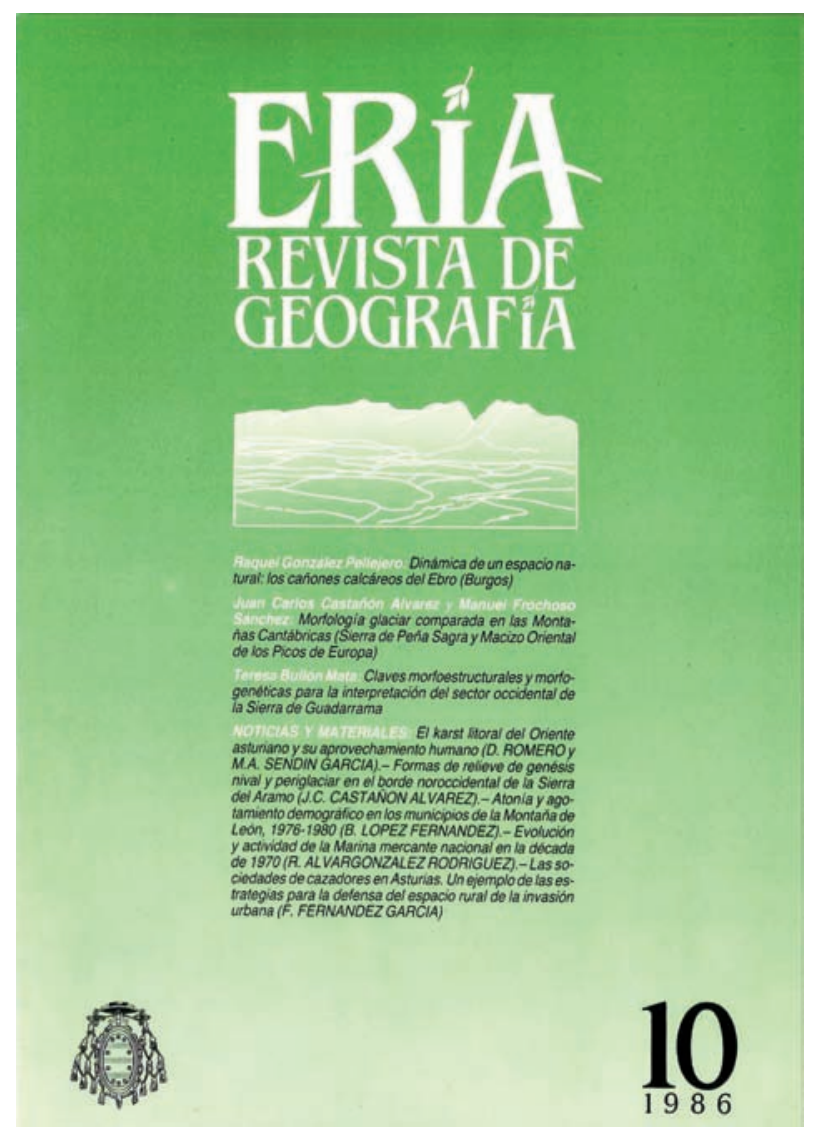

Portada del número 10 de la revista, que cambiaba el aspecto exterior e incluía algunas modificaciones en el interior.

primer momento, aunque tardara un tiempo en alcanzarlo, la pretensión de ir más allá de lo local para abrirse a otros ámbitos y a otros autores con formas similares de entender la Geografía, y su aparición trascendía también lo temporal, pues se insertaba en una línea de pensamiento que, a través del propio Quirós y su estancia en el Instituto Juan Sebastián Elcano del CsIC, en Madrid, conectaba Ería con una forma de concebir la transmisión de los resultados de las investigaciones de los geógrafos.

Así pues, cuando nace Ería no lo hace de la nada, arranca con un legado impagable: la experiencia acumulada por Quirós a lo largo de los diez años durante los que se ocupó de la revista Estudios Geográficos, en estrecha colaboración con don Manuel de Terán. De aquel aprendizaje, de aquellos tiempos de trabajo en Elcano, proceden algunos de los rasgos que han acabado por ser seña de identidad de la revista: la exigencia y el rigor de los trabajos publicados, el cuidado en la presentación formal y, sobre todo, en mi opinión, el mimo con el que se ha tratado la representación gráfica, inculcando a los

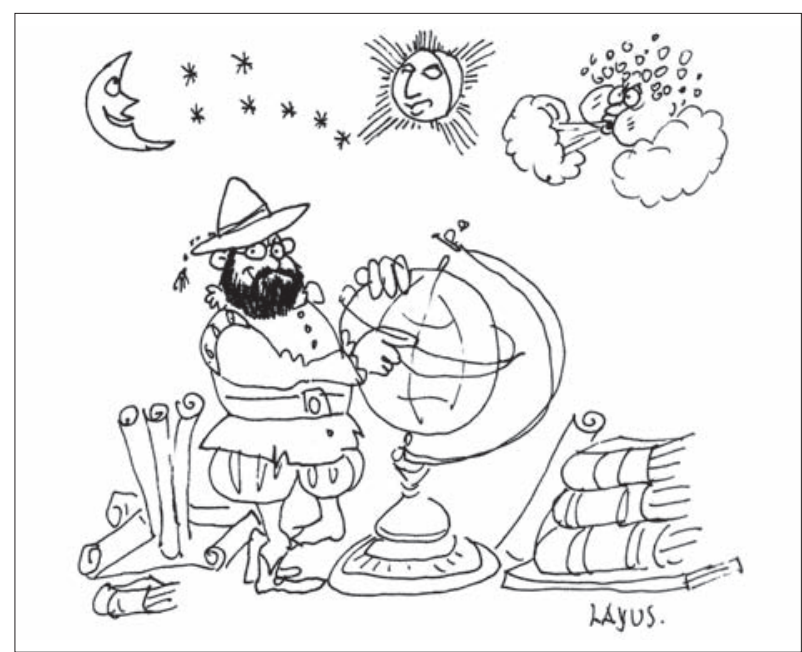

Don Francisco Quirós Linares en una viñeta dibujada por Eduardo Martínez de Pisón a comienzos de los años noventa.

que hemos sido sus discípulos su importancia, que hemos acabado interiorizando y que hemos tratado de trasladar a todas nuestras publicaciones, conformando el conjunto, sin pretenderlo, un auténtico «libro de estilo»; pero en este terreno la revista se ha ocupado de otra tarea no menos importante, la de adaptar la producción gráfica del conjunto de las aportaciones al estilo propio que Ería fue definiendo, y en esta labor la colaboración de José Luis Seoane ha sido determinante.

Durante sus primeros años de vida la revista se nutrió básicamente de las investigaciones del propio Quirós, de las del primer grupo de discípulos que le acompañaron en su traslado desde la Universidad de La Laguna, de las de los geógrafos físicos que se fueron añadiendo al grupo procedentes de Madrid, y finalmente, de las de los que nos fuimos formando bajo su tutela en la propia universidad ovetense, con la colaboración ocasional de geógrafos de otras universidades muy identificados, en lo profesional, y en lo personal y afectivo, con Quirós, como fue el caso de Eduardo Martínez de Pisón.

Pero al igual que les ocurriera a las erías cerealistas en los siglos XVIII y XIX, que debieron adaptar sus prácticas, y también su aspecto formal en parte, a la incorporación de nuevos cultivos, nuestra Ería, al tiempo que cambiaba su aspecto formal, fue incorporando a su nómina de colaboradores investigadores de otras universidades, de manera que en el número 11 (1986) la cantidad de autores no pertenecientes al Departamento de Oviedo superaba por primera vez a la de los de «la casa».

Poco a poco la revista se fue consolidando, hasta alcanzar el punto de madurez que ahora tiene, aunque no se 


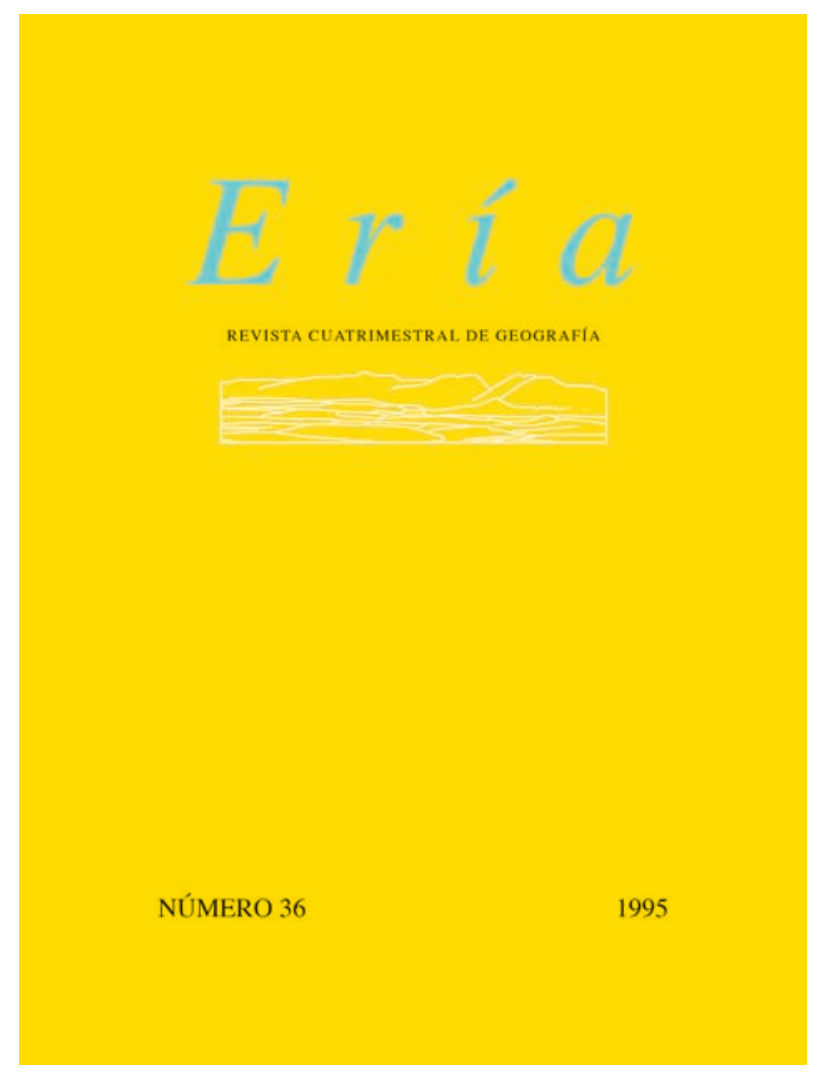

Portada del número 36 de la revista, que inauguraba el formato, tanto interior como exterior, que se ha mantenido hasta la actualidad. vea refrendado por algunos criterios de clasificación de revistas científicas, y en ese recorrido la labor del fundador de la revista, más allá de las cuestiones arriba apuntadas, fue determinante; difícilmente podría expresar yo lo que supuso tal labor mejor que lo hizo nuestra compañera Amalia Maceda con las palabras que escribía en la presentación del libro que la Universidad de Oviedo editó en 2006 como homenaje al profesor Quirós con motivo de su jubilación:

\footnotetext{
Imposible imaginar entonces que aquel sencillo y artesanal «instrumento colectivo», como la concibió su director («Presentación», Ería n. ${ }^{\circ} 1,1980$ ), pudiera llegar al estadio de madurez que ha alcanzado y a convertirse, veinticinco años más tarde, tal vez en la mejor revista geográfica del país. No es lugar este para detenernos en detallar las incomprensiones y los obstáculos que en más de una ocasión amenazaron seriamente su continuidad, pero sí lo es para reconocer que si Ería superó las dificultades fue gracias al convencimiento de don Francisco Quirós sobre su utilidad y su necesidad, y a su empeño en consolidar la que para él y para el resto de los profesores del Departamento es «la revista». No ahorró para ello gestiones, negociaciones ni argumentos ni, porque lo creyó de justicia, se dejó en el tintero la denuncia, en forma de advertencia inserta en varios números: «esta revista no está subvencionada por ninguna entidad pública ni privada, y se publica a costa del profesorado de la Sección de Geografía de la Universidad de Oviedo.

Quien concibió, creó, mantuvo contra viento y marea, dirigió y perfeccionó la revista hasta hacer de ella lo que es hoy Ería, ha de sentirse satisfecho con su obra, aun cuando esa satisfacción ha estado y sigue estando preñada de una intranquilidad permanente por el resultado final de cada nuevo número.
}

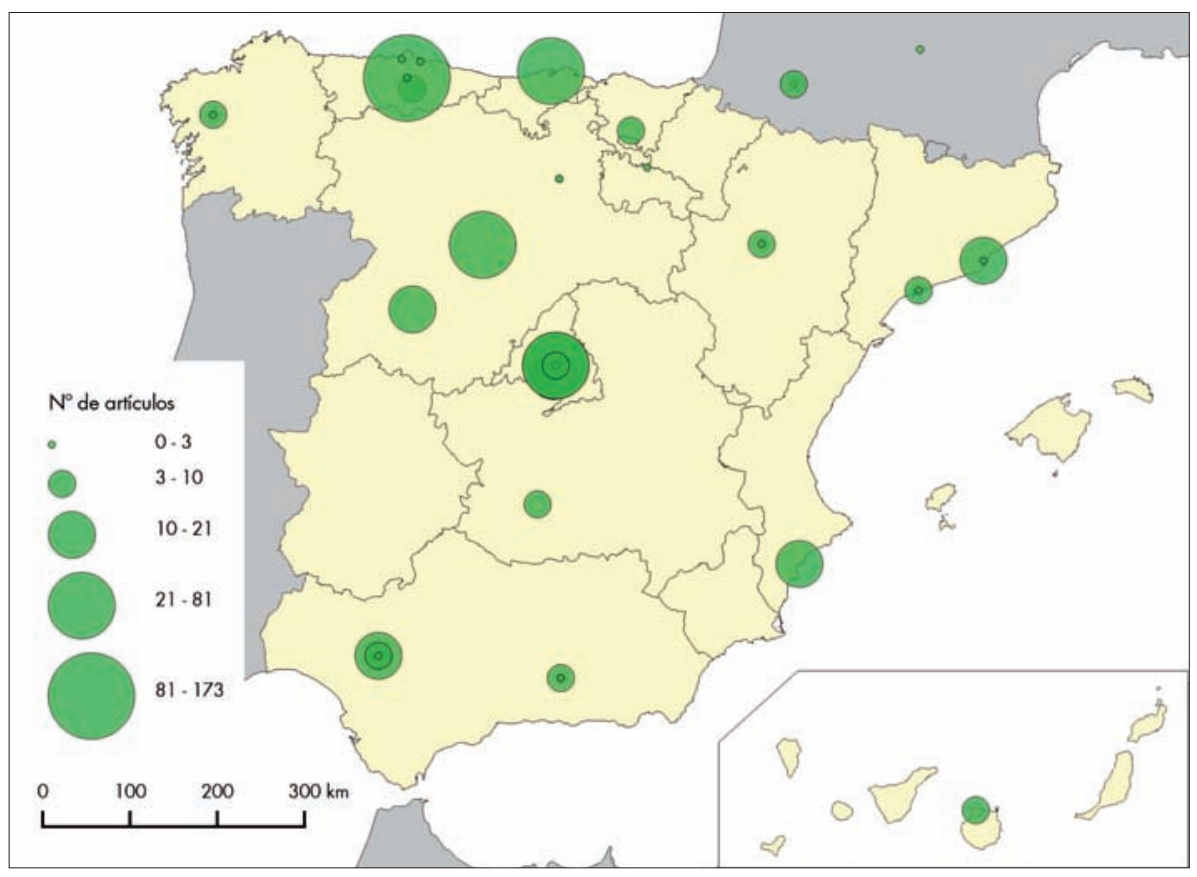

Centro de tra-bajo de los autores que han publicado 


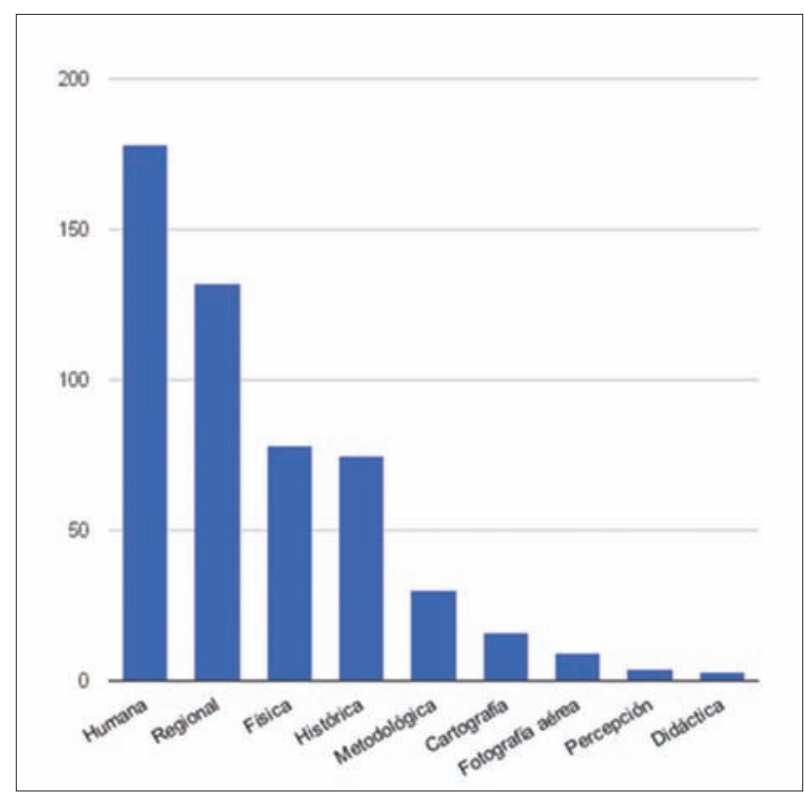

Número de publicaciones, catalogadas por grandes ramas de la Geografía.

\section{LA REVISTA EN CIFRAS}

El $26 \%$ de las autorías de los 525 artículos publicados en Ería corresponde a investigadores de la Universidad de Oviedo, concentrándose su aportación, como ya se ha apuntado, principalmente en los primeros años de vida de la revista; ocupan un lugar relevante a continuación las contribuciones de investigadores de universidades que han tenido históricamente vinculación con la revista, los responsables de cuyos departamentos han formado parte activamente de los órganos de Ería, llegando al punto, en algún caso, de compartir durante unos años la dirección con Francisco Quirós.

Cabe destacar también que el afán de la revista de abrirse progresivamente a colaboraciones de fuera de Asturias, o no vinculadas con la Universidad de Oviedo, se ha plasmado en las aportaciones de un total de 77 instituciones, de las que 23 fueron extranjeras, sumando estas últimas 34 autorías.

En lo tocante a las temáticas abordadas, los trabajos de Geografía Humana, Regional y Física han concentrado, por ese orden, el grueso de los asuntos publicados en Ería; pero un repaso al conjunto pone de manifiesto algo importante: la relevancia que han tenido en la revista tres asuntos que han sido especialmente cuidados por el Departamento de Geografía de Oviedo y que han sido altamente valorados en la línea editorial: la Geografía Histórica, la Cartografía y la Fotografía Aérea.

Por lo que se refiere a los ámbitos geográficos estudiados, Asturias ha sido la provincia con mayor número de trabajos publicados sobre su territorio, pero es bien

Distribución territorial de los asuntos abordados en los artículos publicados en la revista.

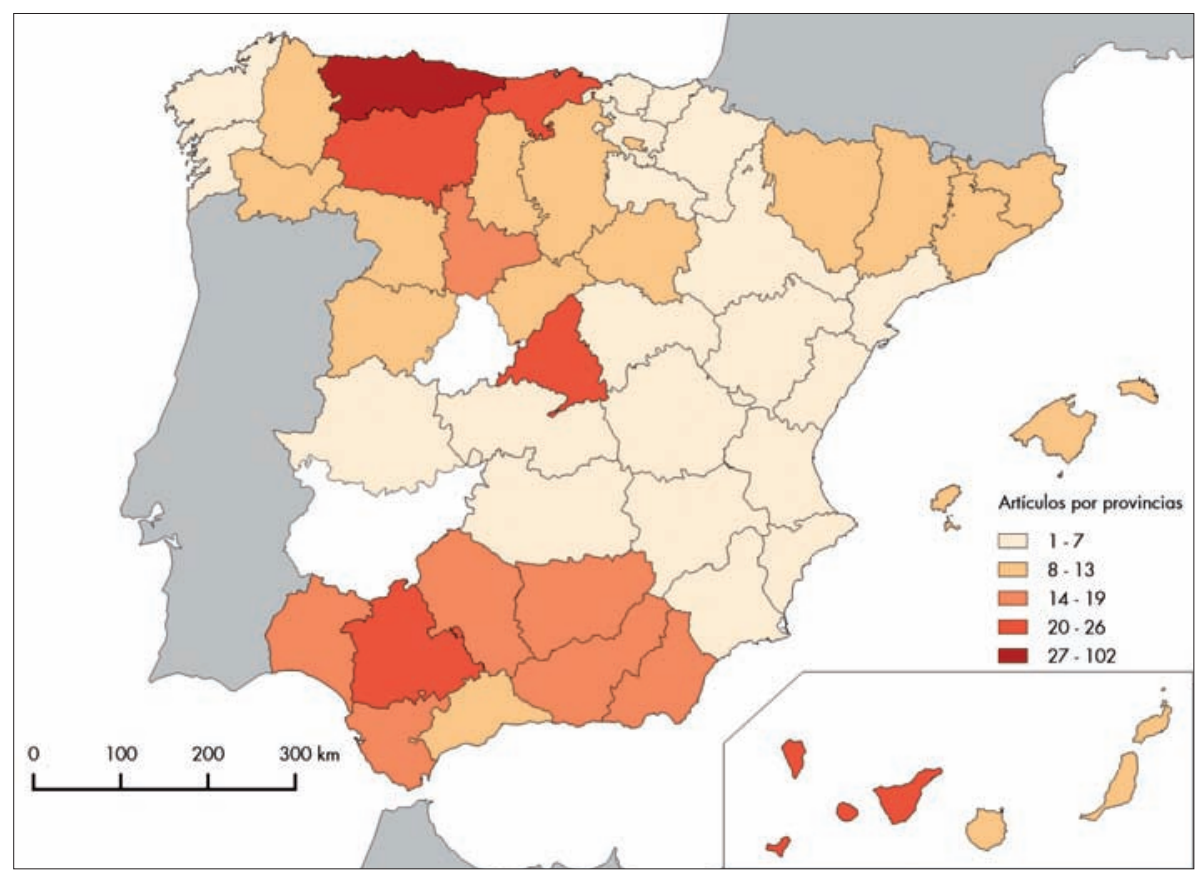




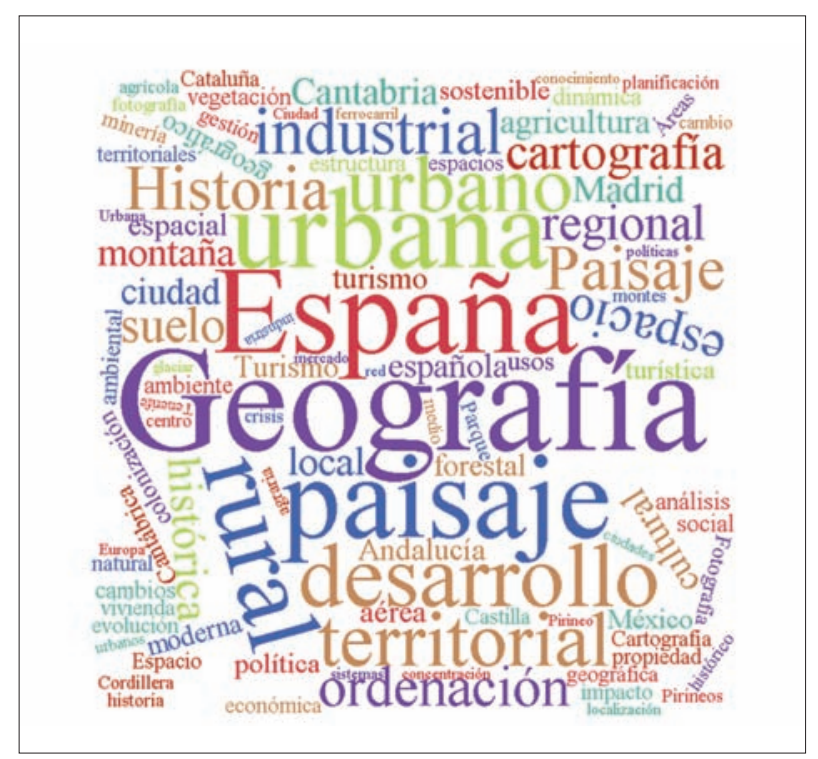

Nube de palabras elaborada a partir de la frecuencia de las palabrasclave de los artículos publicados.

revelador del afán de apertura que ha guiado la trayectoria de la revista que estos solo suponen un $18 \%$ del total. Ocupan los siguientes lugares los referidos a las vecinas provincias de León y Comunidad de Cantabria, o a ámbitos en los que los investigadores de sus universidades han colaborado muy activamente con Ería, como Madrid, Sevilla o Santa Cruz de Tenerife.

Durante los tres primeros años la revista tuvo periodicidad anual; a partir de 1983, y hasta 1985, paso a ser semestral; y desde 1986 hasta la actualidad se han venido publicando con regularidad tres números cada año. Desde su número 15 incluye después de los resúmenes de los artículos un repertorio de «palabras clave»; de su análisis se puede extraer alguna conclusión referida a lo que ha sido la línea editorial: el término «Geografía» es el que más veces aparece, acompañado unas veces de su concreción administrativa («España» en primer y destacadísimo lugar, seguido de algunas comunidades autónomas, y de algún país extranjero), o de su referencia temática («paisaje», «urbano», «rural»...).

\section{PRESENTE Y FUTURO DE LA REVISTA ERÍA}

Este número especial de la revista Ería, con el que cerramos un ciclo, quiere ser un homenaje a su creador, nuestro maestro Francisco Quirós, y también un homenaje al Departamento que él fundó. Han publicado en él la práctica totalidad de los que somos o han sido en algún momento profesores del Departamento; el nivel de los trabajos y la calidad de la representación gráfica, son, a mi juicio, una muestra de madurez, y seguro que cuando Quirós hojee este grueso ejemplar, con esa habilidad suya bien conocida por algunos de detectar al vuelo las erratas, o los disparates, encontrará no pocas faltas que, pese al cuidado puesto en la edición, se nos habrán escapado; pero seguro que, en algún momento - creo y espero que muchos - verá en algún texto, en algún mapa, la huella, más profunda de lo que él cree, que nos ha dejado.

Quiero mostrar mi agradecimiento a todos los autores por su generosidad, y también a José Luis Seoane por el cuidado con el que ha revisado la parte gráfica, y por la valiosa ayuda prestada en la tarea de coordinación de un número de la revista tan complejo; de igual modo hago extensivo el agradecimiento a José Antonio Martín, responsable de la maquetación de Ería desde hace ya muchos años, y a Ceferino y Nicanor, responsables de la imprenta Ápel, también viejos compañeros de faenas en esta aventura que ha sido la publicación de la revista, por el interés que todos han puesto para que la edición de este número estuviera a la altura de la persona a la que va dedicado.

Pero Ería no se detiene; sencillamente cambia de apariencia, sin renunciar a ninguno de los elementos que han constituido sus señas de identidad. Será una revista electrónica, pues no queda otra salida que adaptarse a los nuevos canales de difusión de las publicaciones científicas, pero mientras quede uno sólo de los que apreciamos la publicación en papel, Ería seguirá apareciendo puntualmente en su formato tradicional.

Felipe Fernández García 
Cuadro I. Centro de trabajo de los autores que han publicado en Ería

\begin{tabular}{|c|c|c|c|}
\hline INSTITUCIÓN & APORTACIONES & INSTITUCIÓN & APORTACIONES \\
\hline Universidad de Oviedo & 173 & Universidad Autónoma de Barcelona & 1 \\
\hline Universidad Autónoma Madrid & 81 & Universidad de Burgos & 1 \\
\hline Universidad de Cantabria & 42 & Universidad de Cádiz & 1 \\
\hline Universidad Complutense Madrid & 30 & Universidad de les Illes Balears & 1 \\
\hline Universidad de Valladolid & 27 & Universidad Jaume I de Castellón & 1 \\
\hline Universidad de La Laguna & 21 & Universidad de La Rioja & 1 \\
\hline Universidad de Sevilla & 19 & Universidad de Bergen & 1 \\
\hline Universidad de Salamanca & 18 & Universidad de Buenos Aires & 1 \\
\hline Universidad de Córdoba & 16 & Universidad de Guadalajara. México & 1 \\
\hline Universidad de Alicante & 15 & Universidad Michel-de-Montaigne-Bordeaux & 1 \\
\hline Universidad de Barcelona & 15 & Universidad Nacional Autónoma de México & 1 \\
\hline Universidad de Valencia & 12 & Universidad Nacional de Cuyo. Mendoza & 1 \\
\hline Universidad Carlos III & 11 & Universidad París - I & 1 \\
\hline Universidad de León & 10 & Universidad de Vechta (Deutschland) & 1 \\
\hline Universidad de Santiago de Compostela & 8 & Institut Arbeit und Technik. NRW & 1 \\
\hline UPV - EHU (País Vasco) & 7 & Colegio Universitario de Burgos & 1 \\
\hline Universidad Pablo de Olavide & 7 & Colegio Universitario de La Rioja & 1 \\
\hline Universidad de Zaragoza & 7 & Escuela Nacional de Antropología Social de México & 1 \\
\hline UNAM México & 7 & Geología Aplicada y Medioambiente, S. L. & 1 \\
\hline Universidad de Granada & 6 & Ingeniería $75, \mathrm{~S} . \mathrm{A}$. & 1 \\
\hline Universidad de Las Palmas de Gran Canaria & 6 & IES Cerdeño & 1 \\
\hline Universidad de Lleida & 6 & IES Leonardo Da Vinci. Madrid & 1 \\
\hline Universidad de Castilla-La Mancha & 5 & Instituto de Geología Económica & 1 \\
\hline CECODET & 5 & Muséu del Pueblu d'Asturies & 1 \\
\hline CSIC & 5 & Sociedade galega de Xeografia & 1 \\
\hline Universidad de Extremadura & 4 & UMR Géographie-cité équipe EHGo Paris & 1 \\
\hline Universidad de Girona & 4 & University of Western Australia & 1 \\
\hline Universidad de Jaén & 4 & IES «Virgen de la Caridad», Loja, Granada & 1 \\
\hline Universidad Politécnica Valencia & 4 & IES «Jiménez de Quesada», Santa Fe, Granada & 1 \\
\hline Universidad Rovira i Virgili & 4 & Universidad de Toulouse-Le Mirail & 1 \\
\hline Universidad de La Habana & 4 & Universidad de Pau & 1 \\
\hline Université de Pau et des Pays de l'Adour & 4 & Universidad Joseph Fourier (Grenoble) & 1 \\
\hline Universidad de Málaga & 3 & Universidad de Tarragona & 1 \\
\hline Universidad Politécnica de Madrid & 3 & Saint Louis University & 1 \\
\hline Universidad París - VII & 3 & El Colegio de Michoacán, A. C. & 1 \\
\hline Universidad de Artois & 2 & CONICET & 1 \\
\hline CNRS - Francia & 2 & Instituto Geográfico Nacional & 1 \\
\hline Instituto Pirenaico de Ecología (Zaragoza) & 2 & Centro de Estudios Paisaje y Territorio & 1 \\
\hline Université Michel de Montaigne Bordeaux & 2 & Universidad Hassan II de Casablanca & 0 \\
\hline Colegio San Fernando (Avilés) & 2 & & \\
\hline
\end{tabular}


CuADro II. Distribución territorial de los asuntos abordades en los artículos

\begin{tabular}{|c|c|c|c|c|c|c|}
\hline \multicolumn{2}{|l|}{ POR PROVINCIAS } & \multirow{2}{*}{$\begin{array}{c}\begin{array}{c}\text { VALORES } \\
\text { ORDENADOS }\end{array} \\
102\end{array}$} & \multirow{2}{*}{$\frac{\text { POR PROVINCIAS }}{\text { Lleida }}$} & \multirow{2}{*}{$\begin{array}{c}\begin{array}{c}\text { VALORES } \\
\text { ORDENADOS }\end{array} \\
12\end{array}$} & POR PROVINCIAS & \multirow{2}{*}{$\begin{array}{c}\begin{array}{c}\text { VALORES } \\
\text { ORDENADOS }\end{array} \\
5\end{array}$} \\
\hline Asturias & & & & & Guipuzkoa & \\
\hline Sevilla & & 26 & Soria & 12 & La Rioja & 5 \\
\hline Cantabria & & 25 & Ávila & 11 & Cáceres & 4 \\
\hline León & & 25 & Girona & 11 & Toledo & 4 \\
\hline Madrid & & 25 & Málaga & 11 & Álava & 3 \\
\hline Almería & & 21 & Barcelona & 10 & Ciudad Real & 3 \\
\hline Santa Cruz de Tene & erife & 21 & Palencia & 10 & Cuenca & 3 \\
\hline Córdoba & & 19 & Segovia & 10 & Bizkaia & 3 \\
\hline Valladolid & & 17 & Lugo & 9 & Zaragoza & 3 \\
\hline Cádiz & & 16 & Ourense & 9 & Navarra & 2 \\
\hline Granada & & 16 & Zamora & 9 & A Coruña & 1 \\
\hline Huelva & & 16 & Pontevedra & 7 & Albacete & 1 \\
\hline Jaén & & 16 & Tarragona & 7 & Castellón & 1 \\
\hline Burgos & & 13 & Alicante & 6 & Murcia & 1 \\
\hline Huesca & & 13 & A Coruña & 6 & Teruel & 1 \\
\hline Las Palmas & & 13 & Valencia & 6 & & \\
\hline \multirow[t]{16}{*}{ Salamanca } & & 13 & Guadalajara & 5 & & \\
\hline & POR & PAÍSES & VALORES ORDENADOS & POR PAÍSES & VALORES ORDENADOS & \\
\hline & México & & 10 & Andorra & 1 & \\
\hline & Marruecos & & 9 & Australia & 1 & \\
\hline & Argentina & & 8 & Canadá & 1 & \\
\hline & Cuba & & 7 & Colombia & 1 & \\
\hline & Francia & & 5 & Dinamarca & 1 & \\
\hline & Antártida & & 2 & Ecuador & 1 & \\
\hline & Argelia & & 2 & Estados Unidos & 1 & \\
\hline & Chile & & 2 & Haití & 1 & \\
\hline & Guatemala & & 2 & Inglaterra & 1 & \\
\hline & Noruega & & 2 & Israel & 1 & \\
\hline & Pakistán & & 2 & Nepal & 1 & \\
\hline & Perú & & 2 & Nicaragua & 1 & \\
\hline & Albania & & 1 & Paraguay & 1 & \\
\hline & Alemania & & 1 & República Dominicana & 1 & \\
\hline
\end{tabular}

